\title{
STUDY ON EXERCISE TOLERANCE AND POST EXERCISE DIASTOLIC FILLING PATTERN IN PATIENTS WITH RESTING GRADE 1 DIASTOLIC DYSFUNCTION
}

\author{
Saravanababu Sundararaman ${ }^{1}$, Kumaran Srinivasan ${ }^{2}$
}

${ }_{1}^{1}$ Assistant Professor, Department of Cardiology, Madras Medical College.

${ }^{2}$ Assistant Professor, Department of Cardiology, Madras Medical College.

\section{BACKGROUND}

ABSTRACT

Diastolic dysfunction is one of the commonly seen abnormalities in heart disease patients. It is most commonly associated with diabetes, hypertension, left ventricular hypertrophy, increasing age, coronary artery disease and may even be seen in patients with normal systolic function. This study is about exercise tolerance and post exercise diastolic filling pattern in patients with resting grade-1 diastolic dysfunction.

\section{MATERIALS AND METHODS}

The study population consisted of 104 patients attending outpatient section of cardiology department, Madras medical college and Rajiv Gandhi government general hospital, Chennai. Sample size was taken conveniently

\section{RESULTS}

The most important observation is that in group 1 subjects E/A ratio is increased and in group 2 subjects E/A ratio is decreased

\section{CONCLUSION}

This study highlights the functional capacity/exercise tolerance among patients with Grade-1 diastolic dysfunction at rest showed a heterogenous pattern. The post exercise transmitral Doppler changes showed divergent patterns which can aid in evaluating degree of diastolic dysfunction.

\section{KEYWORDS}

Grade1 Diastolic Dysfunction, Exercise Tolerance, Post Exercise Diastolic Filling Pattern.

HOW TO CITE THIS ARTICLE: Sundararaman S, Srinivasan K. Study on exercise tolerance and post exercise diastolic filling pattern in patients with resting grade 1 diastolic dysfunction. J. Evolution Med. Dent. Sci. 2018;7(09):1144-1147, DOI: $10.14260 /$ jemds/2018/260

\section{BACKGROUND}

Normal ventricular diastolic function is the ability of the ventricles to relax after ejection and to allow adequate filling to a normal end diastolic volume during rest and exercise without abnormal increase in left ventricular diastolic pressure and left atrial pressure.

Diastolic dysfunction is defined as the impaired capacity of the ventricles to accept blood to fill without a compensatory increase of atrial pressure.

Determinants of diastolic function are relaxation of the myocardium, preload, afterload, dyssynchrony of myocardial function, myocardial stiffness, chamber geometry and wall thickness.

\section{Evaluation of Diastolic Function}

Echocardiography, radio-nuclide studies, MRI and Invasive studies are used to evaluate diastolic function. Echo is simpler, cheaper and widely available. Various echo tools for diastolic assessment are M-mode measurement, 2D echo measurement, Doppler measurement-transmitral Doppler measurement, pulmonary venous flow measurement, colour

'Financial or Other Competing Interest': None.

Submission 12-09-2017, Peer Review 10-02-2018,

Acceptance 17-02-2018, Published 26-02-2018.

Corresponding Author:

Dr. Saravanababu Sundararaman,

Flat No. A-704, The Atlantic,

No. 3, Montieth Road, (Red Cross Road),

Egmore, Chennai-600008, Tamilnadu.

E-mail:dr_sarbabu@yahoo.com

DOI: $10.14260 /$ jemds $/ 2018 / 260$

\section{(c) $(i) \$$}

M mode, tissue Doppler imaging methods. Echo Doppler parameters of diastolic function are isovolumic relaxation time, mitral inflow parameters.

\section{Types of Diastolic Filling Patterns in Diastolic} Dysfunction

1. Normal pattern.

2. Grade 1 .

3. Grade 2 (Pseudonormalisation).

4. Grade 3 (Reversible filling pattern).

5. Grade 4 (Irreversible filling pattern).

In normal pattern $\mathrm{E}$ wave is greater than $\mathrm{A}$ wave resulting in $\mathrm{E} / \mathrm{A}>1.5$, deceleration time is $160-240 \mathrm{msec}, \mathrm{E} / \mathrm{E}^{\prime}<8$. In grade-1 diastolic dysfunction, filling pressures are normal $\mathrm{E} / \mathrm{A}<1$, deceleration time $>240 \mathrm{msec}, \mathrm{E} / \mathrm{E}^{\prime}>8$.

Clinical assessment of diastolic function assessment is for filling pressure assessment during rest and with exercise, clinical assessment of cardiomyopathy, diastolic heart failure, constrictive pericarditis and for prognostic assessment.

\footnotetext{
Aims and objectives

To assess the functional capacity/exercise tolerance of patients, to identify different patterns of Doppler parameters before and after exercise, to assess the association between left ventricular filling pattern post exercise and functional capacity/exercise tolerance in patients with resting grade-1 diastolic dysfunction and to identify subgroup of patients with grade-1 diastolic dysfunction who show increase in left ventricular filling pressures after exercise.
} 
MATERIALS AND METHODS

Study Design

Descriptive comparative study.

\section{Study Period}

March 2016 to February 2017.

\section{Inclusion Criteria}

Group 1. Subjects with resting grade-1 diastolic dysfunction in sinus rhythm with normal resting wall motion and EF $>55 \%$ Group-2. Subjects with normal resting diastolic function in sinus rhythm with normal resting wall motion and $\mathrm{EF}>55 \%$

\section{Exclusion Criteria}

Age $<30$ years $>60$ years, moderate and severe valvular regurgitant lesions, coronary artery disease, chronic diseases like liver, lung disease, pregnancy, arrhythmias, systolic dysfunction, uncontrolled hypertension, inability to perform treadmill test, acute systemic illness, pulmonary embolism, aortic dissection.

\section{Sample Size}

104 patients were screened in cardiology OP of which 52 were excluded (52 cases \&52 controls) were selected after applying inclusion criteria. An informed written consent was obtained from all the study patients. Sample size was taken conveniently.

Resting and exercise echo was done using Esaote echo machine, exercise stress testing was performed using Schiller treadmill and standard Bruce protocol was used. Post exercise, subjects were asked to lie down within 45 to 60 seconds and echo was done in parasternal long axis, apical four chamber, five chamber and subcostal views. Left ventricular end diastolic volume, left ventricular systolic volume were obtained at rest and post exercise using biplane Simpson's method.

Using transmitral Doppler indices peak rapid filling velocity (E), peak atrial filling velocity (A) were obtained. Data was analysed by SPSS statistical software and $p$ value of $<0.05$ was considered significant, $p$ value of $<0.01$ was taken as more significant. Discrete variables are sex, diabetes, LVH. Continuous variables are age, heart rate, BP, E-velocity, A-velocity, IVRT, DT, RFP, AFP, E/e'. Continuous variables were described as mean+ or - SD. For comparison between discrete variables chi-square test was used. For comparing discrete with continuous variables student $t$ test was used.

\section{RESULTS}

\begin{tabular}{|c|c|c|c|}
\hline Characteristics & $\begin{array}{c}\text { Group Number I } \\
\text { (Percentage) }\end{array}$ & Group II & P Value \\
\hline Age(years) & $49.3 \pm 5.9$ & $47.6 \pm 6.9$ & 0.18 \\
\hline Male & $35(67.3)$ & $40(76.9)$ & 0.27 \\
\hline Female & $17(32.7$ & $12(23.1)$ & 0.27 \\
\hline Diabetes mellitus & $23(44.2)$ & $0(0)$ & $<0.001$ \\
\hline $\begin{array}{c}\text { Systemic } \\
\text { Hypertension }\end{array}$ & $32(61.5)$ & $0(0)$ & $<0.001$ \\
\hline $\begin{array}{c}\text { Left Ventricular } \\
\text { Hypertrophy }\end{array}$ & $31(59.6)$ & $0(0)$ & $<0.001$ \\
\hline \multicolumn{4}{|c|}{ Table 1. Characteristics of the study subjects } \\
\hline
\end{tabular}

In this study, study subjects were age and sex matched.

\begin{tabular}{|c|c|c|c|}
\hline Characteristics & $\begin{array}{c}\text { Group I } \\
\text { Mean } \pm \text { SD }\end{array}$ & $\begin{array}{c}\text { Group II } \\
\text { Mean } \pm \text { SD }\end{array}$ & P Value \\
\hline $\begin{array}{c}\text { Resting Heart Rate } \\
\text { (per minute) }\end{array}$ & $76 \pm 5.3$ & $75.7 \pm 5.1$ & 0.27 \\
\hline $\begin{array}{c}\text { Resting SBP } \\
\text { (mm Hg) }\end{array}$ & $126.4 \pm 8.8$ & $118.3 \pm 6.09$ & $<0.001^{*}$ \\
\hline $\begin{array}{c}\text { Resting DBP } \\
\text { (mm Hg) }\end{array}$ & $81.3 \pm 5.1$ & $71.0 \pm 3.0$ & $<0.001^{*}$ \\
\hline $\begin{array}{c}\text { Ejection Fraction } \\
\text { (\%) }\end{array}$ & $61.6 \pm 4.3$ & $61.1 \pm 4.6$ & 0.52 \\
\hline E/A & $0.74 \pm 0.03$ & $1.35 \pm 0.02$ & $<0.001^{*}$ \\
\hline $\begin{array}{c}\text { Deceleration Time } \\
\text { (milliseconds) }\end{array}$ & $263.7 \pm 16.8$ & $190.9 \pm 11.7$ & $<0.001^{*}$ \\
\hline \multicolumn{4}{|c|}{ Table 2. Characteristics of the study subjects } \\
in resting state
\end{tabular}

* - Significant (P Value<0.001)

\begin{tabular}{|c|c|c|c|}
\hline $\begin{array}{c}\text { Doppler } \\
\text { variables }\end{array}$ & $\begin{array}{c}\text { Rest Mean } \\
\pm \text { SD }\end{array}$ & $\begin{array}{c}\text { Exercise } \\
\text { Mean } \pm \text { SD }\end{array}$ & P Value \\
\hline $\begin{array}{c}\text { Heart rate } \\
\text { (beats/min) }\end{array}$ & $76.8 \pm 5.3$ & $148.1 \pm 12.6$ & $<0.001^{* *}$ \\
\hline SBP (mm Hg) & $126.4 \pm 8.8$ & $148.7 \pm 10.8$ & $<0.001^{* *}$ \\
\hline DBP (mm Hg) & $81.3 \pm 5.1$ & $78.65 \pm 7.5$ & $0.045^{*}$ \\
\hline E (cm/sec) & $64.25 \pm 11.8$ & $76.2 \pm 9.8$ & $<0.001^{* *}$ \\
\hline A (cm/sec) & $86.3 \pm 13.2$ & $87.7 \pm 4.26$ & 0.463 \\
\hline E/A & $0.74 \pm 0.03$ & $0.87 \pm 0.13$ & $<0.001^{* *}$ \\
\hline IVRT (millisec) & $87.23 \pm 21.2$ & $68 \pm 24.5$ & $<0.001^{* *}$ \\
\hline DT (millisec) & $263.7 \pm 16.8$ & $262.1 \pm 20.7$ & 0.636 \\
\hline RFP (millisec) & $200.1 \pm 29.7$ & $159.3 \pm 21.5$ & $<0.001^{* *}$ \\
\hline AFP (millisec) & $151.6 \pm 17.5$ & $127.8 \pm 13.5$ & $<0.001^{* *}$ \\
\hline \multicolumn{5}{|c|}{$9.05 \pm 0.35$} & $10.6 \pm 1.9$ & $<0.001^{* *}$ \\
\hline E/e & Table 3. Changes with exercise in subjects with \\
\hline \multicolumn{4}{|c|}{ resting Grade I DD (Group I) } \\
\hline
\end{tabular}

${ }^{* *}$ very significant, *significant

\begin{tabular}{|c|c|c|c|}
\hline $\begin{array}{c}\text { Doppler } \\
\text { Variables }\end{array}$ & $\begin{array}{c}\text { Rest Mean } \\
\pm \text { SD }\end{array}$ & $\begin{array}{c}\text { Exercise } \\
\text { Mean } \pm \text { SD }\end{array}$ & P Value \\
\hline $\begin{array}{c}\text { Heart rate } \\
\text { (beats/min) }\end{array}$ & $75.7 \pm 5.1$ & $164.1 \pm 6.7$ & $<0.001^{* *}$ \\
\hline SBP (mm Hg) & $118.3 \pm 6.1$ & $156.7 \pm 4.6$ & $<0.001^{* *}$ \\
\hline DBP (mm Hg) & $71.1 \pm 3$ & $69.6 \pm 2.9$ & $0.017^{*}$ \\
\hline E (cm/sec) & $73.4 \pm 7.6$ & $94.2 \pm 9.4$ & $<0.001^{* *}$ \\
\hline A (cm/sec) & $54.1 \pm 5$ & $86.6 \pm ` 11.3$ & $<0.001^{* *}$ \\
\hline E/A & $1.35 \pm 0.02$ & $1.09 \pm 0.04$ & $<0.001^{* *}$ \\
\hline IVRT (millisec) & $68.9 \pm 9.9$ & $28.2 \pm 12.6$ & $<0.001^{* *}$ \\
\hline DT (millisec) & $190.9 \pm 11.8$ & $209.7 \pm 14.9$ & $<0.001^{* *}$ \\
\hline RFP (millisec) & $233.6 \pm 19.4$ & $173.9 \pm 25.6$ & $<0.001^{* *}$ \\
\hline AFP (millisec) & $107.8 \pm 16.9$ & $104.7 \pm 10.7$ & 0.235 \\
\hline E/e & $7.04 \pm 0.5$ & $7.34 \pm 0.43$ & $0.02^{*}$ \\
\hline \multicolumn{4}{|c|}{ Table 4. Changes with exercise in subjects } \\
without resting Grade I DD (Group II) \\
\hline
\end{tabular}

${ }^{* *}$ very significant, *significant. 


\begin{tabular}{|c|c|c|c|}
\hline $\begin{array}{c}\text { Doppler } \\
\text { Variables }\end{array}$ & $\begin{array}{c}\text { Rest Mean } \pm \\
\text { SD }\end{array}$ & $\begin{array}{c}\text { Exercise } \\
\text { Mean } \pm \text { SD }\end{array}$ & P Value \\
\hline $\begin{array}{c}\text { Heart rate } \\
\text { (beats/min) }\end{array}$ & $76.8 \pm 5.3$ & $75.7 \pm 5.1$ & 0.274 \\
\hline SBP (mm Hg) & $126.4 \pm 8.8$ & $118.3 \pm 6.1$ & $<0.001^{* *}$ \\
\hline DBP (mm Hg) & $81.3 \pm 5.1$ & $71.1 \pm 3$ & $<0.001^{* *}$ \\
\hline E (cm/sec) & $64.25 \pm 11.8$ & $73.4 \pm 7.6$ & $<0.001^{* *}$ \\
\hline A (cm/sec) & $86.3 \pm 13.2$ & $54.1 \pm 5$ & $<0.001^{* *}$ \\
\hline E/A & $0.74 \pm 0.03$ & $1.35 \pm 0.02$ & $<0.001^{* *}$ \\
\hline IVRT (millisec) & $87.23 \pm 21.2$ & $68.9 \pm 9.9$ & $<0.001^{* *}$ \\
\hline DT (millisec) & $263.7 \pm 16.8$ & $190.9 \pm 11.8$ & $<0.001^{* *}$ \\
\hline RFP (millisec) & $200.1 \pm 29.7$ & $233.6 \pm 19.4$ & $<0.001^{* *}$ \\
\hline AFP (millisec) & $151.6 \pm 17.5$ & $107.8 \pm 16.9$ & $<0.001^{* *}$ \\
\hline E/e & $9.05 \pm 0.35$ & $7.04 \pm 0.5$ & $<0.001^{* *}$ \\
\hline Table 5. Comparison between resting Doppler \\
\multicolumn{4}{|c|}{ variables of Group I and Group II } \\
\hline
\end{tabular}

**very significant.

In group 1, the parameters which showed statistical significance between rest and exercise are E velocity, E/A ratio, IVRT, rapid filling period, atrial filling period and E/e.

In group 2, the parameters which showed statistical significance between rest and exercise are E/A ratio, IVRT, rapid filling period and E/e.

\begin{tabular}{|c|c|c|c|}
\hline $\begin{array}{c}\text { Doppler } \\
\text { Variables }\end{array}$ & $\begin{array}{l}\text { Rest Mean } \pm \\
\text { SD }\end{array}$ & $\begin{array}{l}\text { Exercise } \\
\text { Mean } \pm \text { SD }\end{array}$ & P Value \\
\hline $\begin{array}{c}\text { Heart rate } \\
\text { (beats/min) }\end{array}$ & $148.1 \pm 12.6$ & $164.1 \pm 6.7$ & $<0.001^{* *}$ \\
\hline SBP (mm Hg) & $148.7 \pm 10.8$ & $156.7 \pm 4.6$ & $<0.001^{* *}$ \\
\hline $\mathrm{DBP}(\mathrm{mm} \mathrm{Hg})$ & $78.65 \pm 7.5$ & $69.6 \pm 2.9$ & $<0.001^{* *}$ \\
\hline $\mathrm{E}(\mathrm{cm} / \mathrm{sec})$ & $76.2 \pm 9.8$ & $94.2 \pm 9.4$ & $<0.001^{* *}$ \\
\hline $\mathrm{A}(\mathrm{cm} / \mathrm{sec})$ & $87.7 \pm 4.26$ & $86.6 \pm 11.3$ & 0.516 \\
\hline $\mathrm{E} / \mathrm{A}$ & $0.87 \pm 0.13$ & $1.09 \pm 0.04$ & $<0.001^{* *}$ \\
\hline IVRT (millisec) & $68 \pm 24.5$ & $28.2 \pm 12.6$ & $<0.001^{* *}$ \\
\hline DT (millisec) & $262.1 \pm 20.7$ & $209.7 \pm 14.9$ & $<0.001^{* *}$ \\
\hline RFP (millisec) & $159.3 \pm 21.5$ & $173.9 \pm 25.6$ & $<0.001^{* *}$ \\
\hline AFP (millisec) & $127.8 \pm 13.5$ & $104.7 \pm 10.7$ & $<0.001^{* *}$ \\
\hline $\mathrm{E} / \mathrm{e}$ & $10.6 \pm 1.9$ & $7.34 \pm 0.43$ & $<0.001^{* *}$ \\
\hline \multicolumn{4}{|c|}{$\begin{array}{l}\text { Table 6. Comparison between post exercise Doppler } \\
\text { variables of Group I and Group II }\end{array}$} \\
\hline
\end{tabular}

**very significant.

\begin{tabular}{|c|c|c|c|}
\hline $\begin{array}{c}\text { Doppler } \\
\text { Variables }\end{array}$ & $\begin{array}{c}\text { E/A Increase } \\
\text { with Exercise } \\
\text { Mean } \pm \text { SD } \\
(\mathrm{n}=34)\end{array}$ & $\begin{array}{c}\text { E/A Decrease } \\
\text { with Exercise } \\
\text { Mean } \pm \text { SD } \\
(\mathrm{n}=18)\end{array}$ & P Value \\
\hline $\begin{array}{c}\text { Peak heart } \\
\text { rate change } \\
\text { (beats/min) }\end{array}$ & $70 \pm 21$ & $73 \pm 24$ & 0.68 \\
\hline $\begin{array}{c}\text { Exercise time } \\
\text { (min) }\end{array}$ & $9.4 \pm 1.6$ & $7.2 \pm 1.1$ & $<0.001^{* *}$ \\
\hline $\begin{array}{c}\text { E change } \\
\text { (cm/sec) }\end{array}$ & $+21 \pm 18$ & $-1 \pm 14$ & 0.08 \\
\hline $\begin{array}{c}\text { A change } \\
\text { (cm/sec) }\end{array}$ & $+11 \pm 18$ & $+6 \pm 20$ & 0.62 \\
\hline $\begin{array}{c}\text { E/A change } \\
+0.11 \pm 0.008\end{array}$ & $\begin{array}{c}-0.005 \\
\pm 0.007\end{array}$ & $<0.001^{* *}$ \\
\hline $\begin{array}{c}\text { IVRT change } \\
\text { (metre sec) }\end{array}$ & $-20 \pm 40$ & $-2 \pm 36$ & 0.113 \\
\hline \multicolumn{4}{|c|}{$\begin{array}{l}\text { Table 7. Effects of exercise on trans-mitral Doppler } \\
\text { parameters in subjects with resting Grade I DD }\end{array}$} \\
(Group I) showing either an E/A decrease or increase \\
with exercise
\end{tabular}

\section{DISCUSSION}

In normal persons, with exercise there is an increase in cardiac output. During the initial part, the increase in cardiac output is exercise mediated whereas in the later part the increase in cardiac output is attributed to increase heart rate.

In this study there is significant correlation related to exercise related diastolic dysfunction in patients with diabetes, hypertension, coronary artery disease ${ }^{1-5}$. This finding has been found in other studies also.

There is a strong correlation between E/E' and exercise intolerance. The patients who develop diastolic dysfunction during exercise are those patients who have early exercise intolerance.6-10 Already compensated atria could not further compensate during exercise and hence these patients have relatively early exercise intolerance.

Exercise tolerance is related to diastolic filling patterns. Abnormal filling pattern is related to early exercise intolerance. Another way of analysing exercise tolerance is simply to evaluate exercise intolerance. Shorter the duration, more the exercise intolerance.

Three important echo parameters that had shown a stronger statistical correlation with exercise intolerance are non-shortening of isovolumic relaxation time, shortened deceleration time and continued E/A reversal.11-13

In preexisting diastolic dysfunction, if continue to have $\mathrm{E} / \mathrm{A}$ reversal, there was reduced exercise tolerance and if not continue to have E/A reversal, there was better exercise tolerance. This is consistent with other studies. ${ }^{14}$

In normal diastolic function cases, who developed E/A reversal post exercise also showed reduced exercise tolerance. Limitations of this study is sample population is small and they were predominantly low risk group for coronary artery disease.

\section{CONCLUSION}

This study highlights the functional capacity/exercise tolerance among patients with Grade-1 diastolic dysfunction at rest, showed a heterogenous pattern. The post exercise transmitral Doppler changes showed divergent patterns at rest which can aid in evaluating degree of diastolic dysfunction. There is a clear correlation between the functional capacity/exercise tolerance with post exercise transmitral Doppler changes in both groups. Appearance of $\mathrm{E} / \mathrm{A}$ reversal post exercise in people with previous normal transmitral patterns also have exercise intolerance. Transmitral Doppler pattern at rest and post exercise gives further thoughts about impaired relaxation of left ventricle.

\section{REFERENCES}

[1] Verdecchia P, Schillaci G, Guerrieri M, et al. Prevalence and determinants of left ventricular diastolic filling abnormalities in an unselected hypertensive population. Eur Heart J 1990;11(8):679-91.

[2] Poulsen MK, Henriksen JE, Dahl J, et al. Left ventricular diastolic function in type 2 diabetes mellitus: prevalence and association with myocardial and vascular disease. Circulation Cardiovasc Imaging 2010;3(1):24-31.

\footnotetext{
${ }^{* *}$ very significant.
} 
[3] Szlachcic J, Tubau JF, O'Kelly B, et al. Correlates of diastolic filling abnormalities in hypertension: a Doppler echocardiographic study. Am Heart J 1990;120(2):386-91.

[4] Bryg RJ, Williams GA, Labovitz AJ. Effect of aging on left ventricular diastolic filling in normal subjects. Am J Cardiol 1987;59(9):971-4.

[5] Rydberg E, Willenheimer R, Erhardt L. The prevalence of impaired left ventricular diastolic filling is related to the extent of coronary atherosclerosis in patients with stable coronary artery disease. Coron Artery Dis 2002;13(1):1-7.

[6] Marabotti C, Ebert GA, Palombo C, et al. Relationship of Doppler indexes of left ventricular filling and exercise tolerance. G Ital Cardiol 1992;22(10):1151-6.

[7] Mottram PM, Haluska BA, Marwick TH. Response of Btype natriuretic peptide to exercise in hypertensive patients with suspected diastolic heart failure: correlation with cardiac function, hemodynamics and workload. Am Heart J 2004;148(2):365-70.

[8] Grewal J, McCully RB, Kane GC, et al. Left ventricular function and exercise capacity. JAMA 2009;301(3):286-94.

[9] Burgess MI, Jenkins C, Sharman JE, et al. Diastolic stress echocardiography: hemodynamic validation and clinical significance of estimation of ventricular filling pressure with exercise. J Am Coll Cardiol 2006;47(9):1891-900.
[10] Peteiro J, Pazos P, Bouzas A, et al. Assessment of diastolic function during exercise echocardiography: annulus mitral velocity or transmitral flow pattern? J Am Soc Echocardiogr 2008;21(2):178-84.

[11] Nagueh SF, Appleton CP, Gillebert TC, et al. Recommendations for the evaluation of left ventricular diastolic function by echocardiography: an update from the American Society of Echocardiography. J Am Soc Echocardiogr 2009;22(2):107-33.

[12] Terzi S, Sayar N, Bilsel T, et al. Tissue Doppler imaging adds incremental value in predicting exercise capacity in patients with congestive heart failure. Heart Vessels 2007;22(4):237-44.

[13] Nagueh SF, Middleton KJ, Kopelen HA, et al. Doppler tissue imaging: a noninvasive technique for evaluation of left ventricular relaxation and estimation of filling pressures of pulmonary artery pressure using Doppler velocity time intervals. J Am Col Cardiol 1991;18:1493-8.

[14] Gerdts E, Bjornstad H, Toft S, et al. Impact of diastolic Doppler indices on exercise capacity in hypertensive patients with electrocardiographic left ventricular hypertrophy (a LIFE substudy). J Hypertens 2002;20(6):1223-9. 